\title{
Carotid Plaque Characterization - Comparative Evaluation between USG and Non Contrast MRI in Recently and Remotely Symptomatic Stroke Patients
}

\author{
Rudresh. S. Halawar ${ }^{1}$, Prakash P. ${ }^{2}$, Hirdesh Sahni ${ }^{3}$, Tukaram Rathod ${ }^{4}$, Bhimashankar $\mathbf{N}^{5}$ \\ ${ }^{1}$ Associate Professor, Department of Radio Diagnosis and Imaging, S. Nijalingappa Medical College, Navanagar, Bagalkot, \\ Karnataka, ${ }^{2}$ K. F 305 ETA Star Gardens Apartments, Magadi Road, Bangalore 560023, Karnataka, ${ }^{3}$ Professor \& HOD, \\ Department of Radio Diagnosis and Imaging, Command Hopsital (Air Force), Bangalore, Karnataka, ${ }^{4}$ Assistant Professor, \\ Department of Radio Diagnosis and Imaging, S. Nijalingappa Medical College, Navanagar, Bagalkot, Karnataka, ${ }^{5}$ Senior \\ Resident, Department of Radio Diagnosis and Imaging, S. Nijalingappa Medical College, Navanagar, Bagalkot, Karnataka, \\ India
}

Corresponding author: Dr Rudresh. S. Halawar, Associate Professor, Department of Radio Diagnosis and Imaging, S. Nijalingappa Medical College - Navanagar, Bagalkot, India

DOI: http://dx.doi.org/10.21276/ijcmsr.2020.5.1.30

(c) BY-NC-ND

How to cite this article: Rudresh. S. Halawar, Prakash P., Hirdesh Sahni, Tukaram Rathod, Bhimashankar N. Carotid plaque characterization - comparative evaluation between USG and Non contrast MRI in recently and remotely symptomatic stroke patients. International Journal of Contemporary Medicine Surgery and Radiology. 2020;5(1):A138-A142.

\section{A B S T R A C T}

Introduction: Stroke is one of the leading causes of morbidity and mortality worldwide with age-adjusted prevalence rate of stroke was between $250-350 / 100,000$ in India accounting for $1.2 \%$ of total deaths. Atherosclerotic plaque at the carotid bifurcation is a major cause of ischemic strokes. Study aimed to evaluate the imaging appearances of atheromatous plaque using Ultrasound and MRI.

Material and methods: Sonographic images to be evaluated for Plaque at bifurcation and was classified as homogenous or heterogeneous with proper surface evaluation for irregularity. MRI of the carotids was done and the visualised plaque were grouped into homogenous and heterogeneous categories which in turn were correlated with ultrasound pictures to characterize the type of plaque. The plaque characteristics were then compared with the clinical condition of the patient to compare its relation with the incidence of stroke or TIA.

Results: Ultrasound was found to be more sensitive than MRI for plaque size $<1.5 \mathrm{~mm}$ and to characterize when the area of stenosis was less than $50 \%$.

Conclusion: The study showed that symptomatic individuals had a high degree of correlation with heterogeneous plaques on ultrasound. All heterogeneous lesions on MRI contained intra plaque blood with intracellular methemoglobin. Although MRI gives objectivity to the characterization of the plaque in both recent and remote strokes, ultrasound was found to be more sensitive for plaque disease $<50 \%$ area stenosis

Keywords: Carotid Plaque, Non contrast MRI, USG, Stroke Patients

\section{INTRODUCTION}

Stroke is a serious public health problem and the ischemic stroke accounts for the majority (80\%) of all strokes. Atherosclerotic plaque at the carotid bifurcation is a major cause of ischemic strokes and the vulnerable plaque is associated with ischemic stroke more often than the non vulnerable plaque. ${ }^{1}$

We have imaged carotid bifurcation plaque using US and MRI. These two modalities were used to evaluate 30 plaques each in recently and remotely symptomatic individuals. A comparative evaluation of these two modalities in the two groups of patients was done.

Study aimed to evaluate the imaging appearances of atheromatous plaque using Ultrasound and MRI and to evaluate the differences in imaging between recently symptomatic and remotely symptomatic plaques, Using ultrasound and MRI.

\section{MATERIAL AND METHODS}

This study was conducted at Command Hospital Air Force Bangalore over a period of 22 months from Nov 2009 to Aug 2011. A total of 60 lesions from cases of stroke and TIA who had come to receive treatment in our hospital were taken, which included both patients with both recent and remotely symptomatic ones.

\section{Inclusion criteria}

Only patients and control above the age of 20 were included in the study. 
Recently symptomatic patients are those who have had an episode of ischemic stroke or TIA in the last 02 weeks. Remotely symptomatic patients are those who have had a history of ischemic stroke or TIA attack 3 months before.

\section{Exclusion criteria}

1. Patients who failed to meet the inclusion criteria

2. Patient in whom MRI is contraindicated

3. Patient who are claustrophobic and are afraid to undergo MRI

4. Patients who did not consent for the study.

\section{Imaging protocols}

\section{Carotid Ultrasound and Doppler}

The Ultrasound and doppler equipment available in the department was utilised-Siemens and GE pro logiq 5 with a linear probe with a frequency of 8-12 Mhz.

The color doppler was used to asses the flow within the carotids and also helped in assessing the thickness of the plaque. The carotids were further analysed for their velocities as well as the area and diameter of stenosis.

\section{Interpretation of Data}

1. B mode

- Plaque echogenicity is compared to the echogenicity of sternocleidomastoid muscle in the ultrasound examinations [Hypoechogenic, Isoechogenic and Hyperechogenic, Calcification (Hyperechogenic with shadowing)]

- Maximal thickness of plaque

- Maximal diameter of stenosis and maximal area of stenosis

1. Doppler (blood flow velocity at CCA, ICA and at max stenosis)

\section{MRI Scan}

It was done using class 1.5 Tesla scanner of Siemens (Avanto) and using neck coil with a single channel. Patient was explained the procedure and informed consent was taken. No special patient preparation was required (table-1, 2).

\section{RESULTS}

\section{Age Incidence}

The age of the subjects detected to have carotid bifurcation plaque, in this study ranged from 36 years to 78 years with a mean of $63.45 \pm 10.22$ years $($ Mean \pm SD) The maximum incidence was noted in the 61-70 yrs age group.(41.7\%)

The age of the subjects with recently and remotely symptomatic lesions were within a range of 36 to 77 years, 38 to 78 years with a mean of $61.3 \pm 9.8,65.4 \pm 10.3$ years (Mean $\pm \mathrm{SD}$ ) respectively.

In our study, the maximum incidence of atherosclerotic plaque was between the age group of 61-70 yrs.

Ultrasound, MRI and Plaque

The intimal thickness ranged from $1.1 \mathrm{~mm}$ to $5.6 \mathrm{~mm}$ with a mean of $2.97 \mathrm{~mm}$.

The range of area stenosis in recently and remotely symptomatic lesions had a mean of $41 \%$ and $50 \%$ respectively. In our study, out of the 60 lesions, heterogeneous plaques were associated with symptomatic lesions in $65 \%$ and homogeneous plaques were seen in $35 \%$. Calcification in the carotid bifurcation plaque was not visualized in any of the 60 lesions.

In our study, we were not able to detect the fibrous cap as a separate region of the plaque. Also we did not come across any lesion with surface irregularity suggestive of plaque rupture.

There were 17 (28.3\%) plaques that were homogenous both on ultrasound and MRI. There were 12 (20\%) plaques appeared heterogeneous on both ultrasound and MRI constituted $20.0 \%$. Thus only $48 \%$ of patients showed concordance between US and MRI in regards to homogeneity of the plaque.

More lesions appeared heterogeneous on ultrasound; however the content of these plaques and the stage of blood within the plaque as intracellular methemoglobin could be analyzed only on MRI.

In our study the imaging with gradient recalled image showed poor spatial resolution and the identification of hemorrhage or calcification within the plaque by detecting

\begin{tabular}{|l|c|c|c|c|c|c|c|}
\hline Seq & $\begin{array}{c}\text { FOV Read/ } \\
\text { Phase }\end{array}$ & $\begin{array}{c}\text { Slice thick- } \\
\text { ness }\end{array}$ & $\begin{array}{c}\text { TR/TE } \\
\text { (msecs) }\end{array}$ & Avg & $\begin{array}{c}\text { Flip angle } \\
\text { (Degrees) }\end{array}$ & $\begin{array}{c}\text { Phase encod- } \\
\text { ing direction }\end{array}$ & $\begin{array}{c}\text { Base /Phase } \\
\text { Resolution }\end{array}$ \\
\hline SE T1 & $224 / 100$ & $3 \mathrm{~mm}$ & $486 / 9.2$ & 1 & 150 & $\mathrm{~A} \rightarrow \mathrm{P}$ & $256 / 100$ \\
\hline TSE T2 & $224 / 100$ & $3 \mathrm{~mm}$ & $3670 / 102$ & 1 & 150 & $\mathrm{~A} \rightarrow \mathrm{P}$ & $256 / 80$ \\
\hline SE T1 FS & $224 / 100$ & $3 \mathrm{~mm}$ & $650 / 9$ & 1 & 150 & $\mathrm{~A} \rightarrow \mathrm{P}$ & $320 / 80$ \\
\hline GRE - Medic & $224 / 100$ & $3 \mathrm{~mm}$ & $873 / 29$ & 1 & 20 degrees & $\mathrm{A} \rightarrow \mathrm{P}$ & $256 / 100$ \\
\hline
\end{tabular}

Table-1: MRI Protocol used in our study. SE- Spin Echo,TSE- Turbo spin echo, A $\rightarrow P$ - Anterior to posterior, mm - millimeter, msecs milliseconds

\begin{tabular}{|c|c|c|c|c|}
\hline & Fat & Blood & Calcium & Fibrosis \\
\hline $\mathrm{T} 1$ & 个个 & $\uparrow$ & $\downarrow$ & $\downarrow$ \\
\hline $\mathrm{T} 2$ & $\uparrow$ & $\uparrow \downarrow$ & $\downarrow$ & $\downarrow$ \\
\hline GRE & $\downarrow$ & $\downarrow$ & $\downarrow$ & $=$ \\
\hline T1FS & $\downarrow \downarrow$ & $\uparrow$ & $\downarrow$ & $\downarrow$ \\
\hline Ultrasound & $\downarrow$ & $\downarrow$ & 个个With Shadowing & $\uparrow$ \\
\hline
\end{tabular}


magnetic susceptibility artifact was difficult. It may be due to susceptibility to patient motion, overlapping with the jugular vein, and inability to image carotid stenosis and plaque morphology accurately due to turbulence on GRE images.

In our study we were not able to identify the fibrous cap or its rupture in any of the lesions either on US or MRI.

In our study, the lesions seen in both the sub sets, recently and remotely symptomatic lesions, the plaque morphology was the same and no statistically significant change was noted.

Both recently and remotely symptomatic patients showed heterogeneous lesions more frequently than homogenous lesions. The content of this heterogeneous plaque was intracellular met hemoglobin in all cases which were analyzed on MRI.

The heterogeneous lesions were better visualized on ultrasound as compared to MRI. However the content of these plaques as intracellular met hemoglobin was analyzed only MRI.

\section{DISCUSSION}

Stroke is a serious public health problem in India and Ischemic stroke accounts for the majority (80\%) of all strokes. ${ }^{1}$ Atherosclerosis is a type of arteriosclerosis derived from the Greek words "atheros" meaning "gruel" and "sclerosis" meaning "hardening" and is characterized by intimal lesions called atheromas that protrude into vascular lumina. It is synonymous with atheroma and atheromatous plaque. ${ }^{2}$

Imaging wise plaque is defined as a local thickening of the intimal layer of greater than $1 \mathrm{~mm} .^{3}$

The increased mechanical stress due to high flow velocity in patients with stenosis might lead to intraplaque hemorrhage with subsequent stretching of the intima over the hemorrhage with further turbulent flow and precipitate formation of luminal thrombi or lead to ulceration of intima that stretches over the hemorrhage, all possibly reducing cerebral blood flow by hypo perfusion or embolisation leading to - stroke or transient ischemic attack. ${ }^{4}$

Typical atheromas contain relatively abundant lipid. However, fibrous plaques are composed mostly of smooth muscle cells and fibrous tissue. In advanced atherosclerosis, the fatty atheroma may be converted to a fibrous scar. The complicated lesion of atherosclerosis, defined by the following changes, has the most clinical significance. Focal rupture or gross ulceration or both, of the luminal surface of atheromatous plaques may result in exposure of highly thrombogenic substances that induce thrombus formation (cholesterol emboli or atheroemboli).

- Haemorrhage into a plaque occurs from rupture of either the overlying fibrous cap or the thin walled capillaries that vascularise the plaque.

- Superimposed thrombosis usually occurs on disrupted lesions (those with rupture, ulceration, erosion or hemorrhage).

- Thrombi may partially or completely occlude the lumen; they may become incorporated within the intimal plaque by organization. ${ }^{5-7}$

Carotid artery evaluation using Doppler ultrasound

Zweibel WJ et al, prospectively studied carotid bifurcation and suggested that Doppler and B-mode combined were more accurate than separate use of either procedure for evaluation of occlusive lesions and the level of accuracy in evaluating plaque and stenosis provided a general estimate of severity of disease. ${ }^{8}(45)$

Imparto et al carried out a prospective study on carotid artery plaques which indicated that intramural haemorrhage was the only morphologic characteristic significantly more frequent in symptomatic plaques. ${ }^{9}$

In 1983, Reilly et al, in their study concluded that the heterogeneous plaque was associated with a statistically greater incidence of plaque hemorrhage and ulcerationand was associated with a greater incidence of transient ischemic attack or stroke. ${ }^{10}$

In 1993 Geroulakos et al defined plaques into five types which was adopted and modified by AHA. ${ }^{11}$

Type 1 plaques consisted of uniformly echolucent plaques,

Type 2 plaques consisted of predominantly echolucent plaques with $<50 \%$ echogenic areas,

Type 3 plaques consisted of predominantly echogenic plaques with $>50 \%$ echogenic areas,

Type 4 plaques consisted of uniformly echogenic plaques and Type 5 plaques were those that could not be classified owing to heavy calcification and acoustic shadowing.

The results of a study showed that type 1 plaque was present in $5 \%$ of patient with symptoms and $95 \%$ in those without symptoms there was preponderance of echogenic plaques. ${ }^{11}$

A study by El-Saden et al, stated that MRA- TOF and US could replace DSA if the studies were in agreement (100\% sensitivity and $91 \%$ specificity with an accuracy rate of 94\%), and concluded that agreement with MRA and US meant that further evaluation with DSA was unnecessary. In this study, the statiscal analysis revealed, high sensitivities and specificities when using 3D TOF MRA (100\% sensitivity and $91 \%$ specificity with an accuracy rate of 94\%) but flow gaps resulted in accurate MRA examinations necessitating DSA in only a few patients. ${ }^{12}$

Carotid plaque evaluation by Magnetic resonance imaging Kucharczyk et al in 1994 showed that calcified region of plaque consisted primarily of hydroxyapatite. Due to its low proton density and because of diffusion mediated susceptibility effects calcification was associated with low signal intensity on all the MR sequences. ${ }^{13}$

In 1997 Yuan et al carried out in vitro study to differentiate different types of lipid using T1 weighted (T1W), T2 weighted (T2W) and proton density weighted (PDW) MR images. They showed that among individual lipids, triglycerides, which constituted $6 \%$ of the plaque, had strong signals in all three MR contrast weightings at body temperature. ${ }^{14}$

In a study by Winn et al, a definite reading for detection of fibrous caps or rupture was fairly specific $(90 \%$ and $98 \%$ respectively) but not very sensitive (37\% and $12 \%$ respectively). ${ }^{15}$

Yuan $\mathrm{C}$ et al carried on in vivo study on eighteen patients using 1.5TMR scanner that generated four contrast weighting (T1,T2, proton density and 3D time of flight). Overall accuracy of multispectral MRI was $87 \%$ ( $80 \%$ to 
$94 \%)$ sensitivity was $85 \%$ (78\% to $92 \%)$ and specificity was $92 \%(80 \%$ to $98 \%){ }^{16}$

High signal intensity on T2-SE images was characteristic of lipid, whereas low signal intensity on T2-SE images was characteristic of fibrous tissue. ${ }^{17}$

Yuan et al concluded that identification of a ruptured fibrous cap was highly associated with a recent history of TIA or stroke.

There was a strong and statistically significant trend showing a higher percentage of symptomatic patients with ruptured cap (70\%) and thin cap (50\%) compared with thick cap. ${ }^{18}$ Later in 2004 Chu et al found that MRI detected intrapalque hemorrhage with high sensitivity (90\%) but moderate specificity (74\%). Three stages of intraplaque hemorrhage were categorized: fresh, recent and old. ${ }^{19}$

In another study Kampschulte et al, found that the sensitivity and specificity of MRI to correctly identify cross sections that contained hemorrhage were $96 \%$ and $82 \%$ respectively. ${ }^{20}$ Takaya et al, evaluation twenty nine subjects found that hemorrhage into carotid plaque produced a stimulus for progression of atherosclerosis by increasing lipid core and plaque volume and creating new destabilizing factors. ${ }^{21}$

\section{Limitations of our study}

1. All patients were from service background - age bias, as people while in service are exercising regularly and hence reduced chances of plaque disease.

2. Only patients brought to the hospital were imaged - selection Bias as the hospital caters exclusively people from defence background and their families in and around the location. This does not represent the incidence in the local population.

\section{CONCLUSION}

In our study, a total of 60 lesions from symptomatic individuals were evaluated with ultrasound and MRI. The individuals were further divided into recently and remotely symptomatic. We had analyzed the morphological pattern of the plaque in these two sub sets with ultrasound and MRI and findings were further correlated.

Ultrasound was found to be more sensitive than MRI for plaque size $<1.5 \mathrm{~mm}$. Ultrasound was also better in assessing the plaque characteristics when the area of stenosis was less than $50 \%$. Hence, ultrasound is better as a screening tool.

The study showed that symptomatic individuals had a high degree of correlation with heterogeneous plaques on ultrasound with $66.7 \%$ recently symptomatic individuals showed heterogeneous plaques and the remotely symptomatic patient's individuals had an association of $50 \%$ with a $p$ value of 0.295 .

The homogenous lesions on ultrasound appeared homogenous on MRI with the plaque content for fat. The heterogeneous plaque on ultrasound however was correlated in only $26 \%$ of the MRI. Out of the 39 lesions which appeared heterogeneous on ultrasound only 12 were found to be heterogeneous on MRI with a $\mathrm{p}$ value of 0.345.All heterogeneous lesions on MRI contained intra plaque blood with intracellular methemoglobin.

No cases had calcific plaque. Fibrous cap was not visualized in any of the cases in our study. A thinner slice with a smaller
FOV could have helped in analyzing the fibrous cap.

Although MRI gives objectivity to the characterization of the plaque in both recent and remote strokes, ultrasound was found to be more sensitive for plaque disease $<50 \%$ area stenosis.

\section{REFERENCES}

1. Tapas Kumar Banerjee MD, Shyamal Kumar das DM. Epidemiology of stroke in India Neurology Asia 2006; 11(1): $1-4$.

2. Vinay Kumar,MD; Abul K Abbas, MBBS;Nelson Fausto, MD; Richard Mitchell, MD; Robbins basic pathology $6^{\text {th }}$ edition; Saunders Elsevier:2007 Page 343

3. Carotid ultrasound phenotypes in vulnerable populations Silvia A Riccio, Andrew A House, J David Spence, Aaron Fenster, and Grace Parraga Cardiovasc Ultrasound. 2006; 4(3): 44.

4. Satoki Homma, MD; Nobuyoshi Hirose, MD; Hiroyuki Ishida, MD; Toshiharu Ishii, MD; Goro Araki, MD et al. Carotid Plaque and Intima-Media Thickness Assessed by B-Mode Ultrasonography in Subjects Ranging From Young Adults to Centenarians. Stroke. 2001;32(5):830-835

5. The significance of turbulence in hemic systems and in the distribution of the atherosclerotic lesions Surgery 1965;57(2):155

6. Mc.Gill HG Jr, Strong JP, Natural history of human atherosclerotic lesions: Atherosclerotic and its origin. Academic press, New York, 1963.

7. Roberts AB, Strauss HW, Lees Rs, et al. low density lipoproteins concentrate in Damaged arterial wall (abstr). Circulation 1980;62(111):97.

8. Zweibel WJ, Austin CW, Sackett JF, Strother CM. Correlation of high resolution, B-mode and continuouswave doppler sonography with arteriography in the diagnosis of carotid stenosis, Radiology 1983;149(4):523-532

9. Imparato AM, Riles TS, Mintzer R, Baumann FG. The importance of haemorrhage in the relationship between gross morphologic characteristics and cerebral symptoms in 376 carotid artery plaques Ann surgery 1983;197(6):195-203

10. Reilly LM, Lusby RJ, Hughes L. Ferrell LD, Stoney RJ, Ehrenfeld WK. Carotid plaque histology using real-time ultrasonography. Clinical and therapeutic implications. American Journal of surgery 1983;146(1):188-193

11. Burke GL, Evans GW, Riley WA, et al. The atherosclerosis risk in communities (ARIC) study. Stroke 1995;26(5):386-391

12. El-Saden SM, Grant EG, Hathout GM, Zimmerman PT, Cohen SN, Baker JD, imaging of the internal carotid artery: the dilemma of total versus near total occlusion Radiology 2001;221(3):301-8

13. Kucharczyk W, Henkelman RM. Visibility of calcium on MR and CT; can MR show calcium that CT cannot ? AJNR 1994;15(2):1145-1148

14. Yuan C, Petty C, O’Brien KD, Hatsukami TS, Eary $\mathrm{JF}$, Brown BG. In vitro and in situ magnetic resonance imaging signal features of atherosclerotic plaque associated lipids. Atherioscler otic thromb vasc boil 1997;17(6):1496-1503 
15. Winn WB, Schmiedl UP, Reichenbach DD, et al detection and characterization of atherosclerotic fibrous caps with T2 Weighting MR Am J Neuroradiol 1998;19(4):129-134

16. Yuan C, Mitsumori LM, Ferguson MS, et al In vivo accuracy of multispectral magnetic resonance imaging for identifying lipid rich necrotic cores and intraplaque hemorrhage in advanced human atherosclerotic plaques. Circulation 2001;104(5):2051-2056

17. Fayad ZA, Fuster V, Clinical imaging of the high risk or vulnerable atherosclerotic plaque, Circ Res 2001;89(1):305-316

18. Yuan C, Zhang S, Polissar NL, et al identification of fibrous cap rupture with magnetic resonance imaging is highly associated with recent transient ischemic attack or stroke. Circulation 2002;105(3):181-185

19. Chu. B, Kampschulte A,Ferguson MS, et al Hemorrhage in the atherosclerotic carotid plaque: A high resolution MRI study. Stroke 2004;35(6):1079-1084

20. Kampschulte A, Ferguson MS, Polissar NL, Saam Y, Yuan C, Differentiation of intraplaque versus juxtaluminal hemorrhage / thrombus in advanced human carotid atherosclerotic lesion by invivo magnetic resonance imaging Circulation 2004;110(4):3239-3244

21. Takaya N, Yuan C, Chu B, et al presence of Intraplaque hemorrhage stimulates progression of carotid atherosclerotic plaques. A high resolution magnetic resonance imaging study. Circulation 2005;111(2):27682775

Source of Support: Nil; Conflict of Interest: None

Submitted: 30-11-2020; Accepted: 01-02-2020; Published online: 28-02-2020 